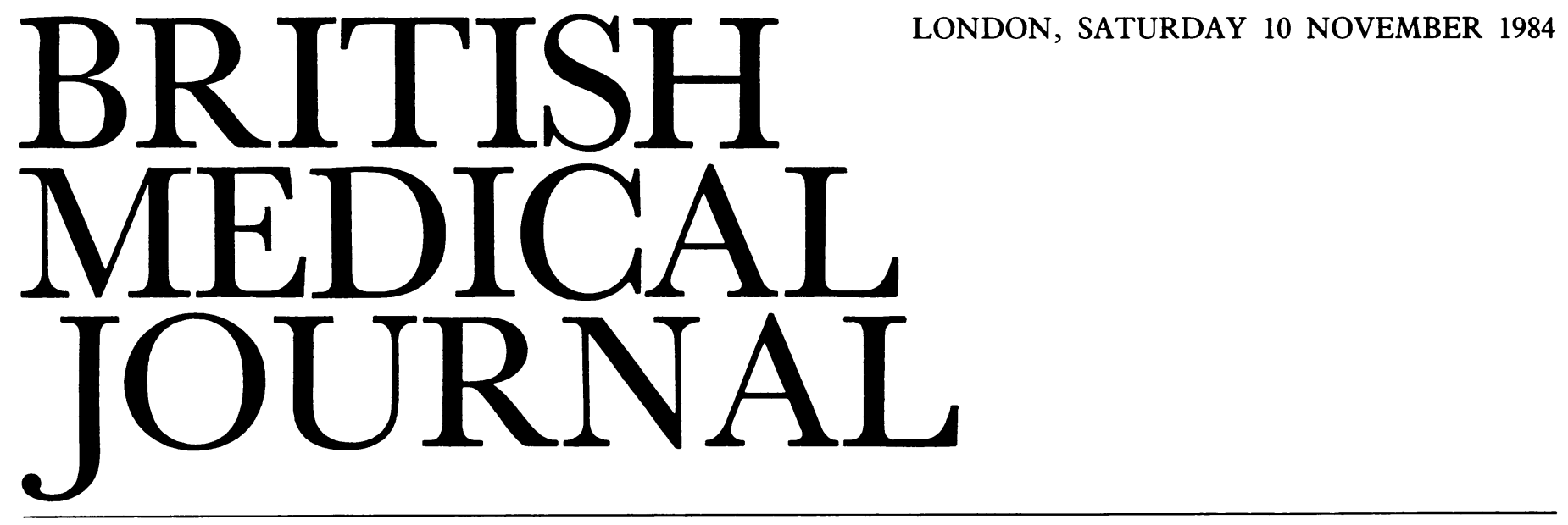

\title{
Who should be immunised against hepatitis B?
}

The public health importance of hepatitis B throughout the world cannot be exaggerated: acute and persistent infection is common, and the incidence is increasing. The world reservoir of hepatitis B carriers is conservatively estimated to number more than 200 million people, and the carrier state may lead to chronic liver disease including chronic active hepatitis, cirrhosis, and hepatocellular carcinoma. Hepatocellular carcinoma is one of the 10 most common tumours in the world, with over 250000 new cases each year, and there is compelling evidence that hepatitis B is its cause in up to $80 \%$ of cases. ${ }^{12}$ Intermediate stages between infection and the development of liver cancer are integration of the virus into the DNA of the host, the establishment of persistent infection, and the carrier state.

The wide range of parenteral and inapparent parenteral routes of transmission of hepatitis B includes the transfusion of blood and certain plasma derivatives; the use of inadequately sterilised syringes, needles, and instruments; and sexual contact, particularly among homosexual men. Hepatitis B is also an occupational hazard among health care and laboratory personnel, and infection may be common in residents and staff of institutions for the mentally handicapped and in custodial institutions. The high rates of transmission of hepatitis B from carrier mothers to their newborn infants in many areas of the world make protective immunisation of susceptible mothers and of infants born to carrier mothers the only practical way of interrupting transmission of the infection and preventing the establishment of the carrier state in most of the infected children.

The development of an unusual vaccine against hepatitis $B$ from the excess surface antigen protein coat of the virus obtained from the plasma of asymptomatic carriers was, therefore, welcome, for it is an ingenious solution to meet the urgent need for an effective vaccine in the face of the repeated failure to grow the virus in tissue culture. The plasma derived vaccine, which was licensed in Britain in the autumn of 1982, has undergone rigorous safety and efficacy studies in the United States and elsewhere. Nevertheless, concern was expressed about the source of the starting plasma, which includes plasma collected from asymptomatic homosexual carriers-giving at least a theoretical risk of contamination of the preparation with the putative agent of the acquired inmune deficiency syndrome (AIDS). This concern soon led to emotional, vociferous, and indeed irrational statements despite reassurance on safety published by the World Health Organisation after review of the accumulated data by several groups of experts. ${ }^{2-4}$

The absurdity of the case against plasma derived vaccine is reinforced by the widespread use of heptatitis B immunoglobulin for passive immunisation. This immunoglobulin is prepared from pooled plasma, a substantial proportion of which is obtained from homosexual men. The immunoglobulin is prepared by Cohn ethanol fractionation and is not subjected to inactivation procedures. A WHO consultative group which met in Geneva in July 1983 considered all the data assembled in order to assess the safety of use of both normal (gammaglobulin) and specific immunoglobulin (such as hepatitis B immunoglobulin). The data provided from six sources concerning immunoglobulins prepared from plasma collected mainly in the United States, including donations from homosexuals, indicated that about 19.5 million doses of 2-10 ml each were prepared between 1979 and 1983 and that recipients were observed for one to four years. There has been no transmission of hepatitis B or any other infectious agent including AIDS by the use of these preparations, and no evidence of risk was attached to the use of immunoglobulins prepared by conventional accepted methods. ${ }^{5}$

The plasma derived hepatitis B vaccine licensed in Britain meets the WHO requirements of 1981 and 1983 and consists of highly purified $22 \mathrm{~nm}$ surface antigen particles which have been subjected to three different inactivation procedures. These procedures have been shown to destroy the infectivity of hepatitis B virus and representatives of all known groups of animal viruses. Thus the purification of hepatitis B surface antigen from plasma entailing isopycnic and rate zonal ultracentrifugation has been shown to be effective in removing $10^{4}$ infectious doses of hepatitis B virus per $\mathrm{ml}$, as determined by the inoculation of susceptible chimpanzees. Treatment with pepsin $1 \mu \mathrm{g} / \mathrm{ml}$ at $\mathrm{pH} 2$ and $37^{\circ} \mathrm{C}$ for 18 hours inactivates $10^{5}$ or more infectious doses of hepatitis $\mathrm{B}$ virus per $\mathrm{ml}$ and has been shown to inactivate virus in the rhabdovirus, poxvirus, togavirus, reovirus, and coronavirus groups. The next step of inactivation is treatment with $8 \mathrm{M}$ urea at $37^{\circ} \mathrm{C}$ for four hours. This process alone inactivates both $10^{5}$ or more infectious doses of hepatitis B virus per $\mathrm{ml}$ and also viruses in the rhabdovirus, myxovirus, poxvirus, togavirus, reovirus, picornavirus, herpes virus, and coronavirus groups. Scrapie virus adapted to mice, representing slow viruses, is inacti- 
vated by $6 \mathrm{M}$ urea. The final step of treatment with formalin inactivates hepatitis $B$ virus, as well as many other viruses, including parvoviruses, retroviruses, and the delta agent. ${ }^{6}$ Human T cell lymphotropic virus (HTLV III or LAV) is a retrovirus and there is strong evidence of a causal association between this virus and AIDS; but as might be expected from the above data AIDS has not been associated with the vaccine.

The hepatitis B plasma derived vaccine, then, meets the WHO requirements revised in 1983 and its safety is now established. The priorities for immunisation against hepatitis $B$ are not the same for each region or country. These needs are dictated by epidemiological patterns, socioeconomic factors, cultural and sexual practices, and the environment. Immunisation against hepatitis B is recommended for six main groups in Britain.

Firstly, health care personnel should be vaccinated if they have frequent contact with blood or needles, if they are staff of residential institutions for the mentally handicapped, take part in direct patient care in units treating carriers, or work in haemodialysis, haemophilia, and other centres providing maintenance treatment with blood or blood products. Dentists and ancillary staff with direct contact with patients, laboratory workers regularly exposed to increased risk from infected material, and personnel on secondment to countries with a high prevalence of hepatitis $B$, if they are directly concerned in patient care, should also be vaccinated. Personnel accidentally pricked with needles used for patients with hepatitis B should be given the vaccine either alone or in combination with hepatitis B immunoglobulin at a contralateral site.

Secondly, the patients who should be vaccinated include first entrants into residential institutions for the mentally handicapped, those treated by maintenance haemodialysis or by frequent transfusion of blood or blood products, those having surgery requiring multiple transfusions or treatment with blood products, or both, and those with chronic renal damage when it appears likely that treatment by haemodialysis or transplantation will ultimately be required.

Thirdly, vaccination should be offered to certain contacts of patients: the sexual partners of patients with acute hepatitis B or carriers and other family members in close contact.

Fourthly, vaccination is recommended for infants born to hepatitis B carriers or $\mathrm{HBsAg}$ positive mothers as a result of recent infections, particularly if e positive or without anti-e. The optimum time for immunisation in combination with hepatitis B immunoglobulin, however, is not yet established.

Fifthly, other groups at risk include staff at reception centres for refugees and immigrants from areas where hepatitis B is very common, such as South East Asia; individuals who frequently change sexual partners-particularly promiscuous male homosexuals and female and male prostitutes; and narcotic drug abusers.

Finally, there are groups at "lower risk," including long term men prisoners; staff of custodial institutions, ambulance, and rescue services; and selected police personnel.

Failure to immunise these people places them at unnecessary and unjustified risk. ${ }^{7}$

ARIE J ZuCKERMAN

Professor of Microbiology and Director of the

WHO Collaborating Centre for Reference and Research on

Viral Hepatitis,

London School of Hygiene and Tropical Medicine,

London WCIE 7HT
1 Zuckerman AJ, Sun T-T, Linsell A, Stjernsward J. Prevention of liver cancer. Report on a WHO scientific group. Lancet 1983;i:463-5.

WHO. Prevention of liver cancer. WHO Tech Rep Ser 1982; No 691

World Health Organisation. Hepatitis programme. Lancet 1983;ii:350

Anonymous. WHO meeting on AIDS. Lancet 1983;ii:1297.

orld Health Organisation. Viral hepatitis. The use of normal and specific immunoglobulin. WHO

Anonymous. Hepatitis B virus vaccine safety: report of an inter-agency group. MMWR Callender ME,

Med f 1982;284:324-6.

\section{Ethics in clinical chemistry}

At first sight there might not appear to be much scope for ethical brooding among the analytical machines and gadgets of a clinical chemistry laboratory. Yet an extensive discussion of ethical issues has in fact been filling sizable portions of the Association of Clinical Biochemists' News Sheet for the past several months.

The inspiration came from a plenary lecture on the subject given in Vienna by BenGershôm at the International Federation of Clinical Chemistry's congress in 1981. Dr BenGershôm is head of the clinical chemistry department at the Sophia Children's Hospital, Rotterdam. His lecture delineated various topics in which, at least by his observation, barriers of professional etiquette deter the clinical biochemist, who may have special skills, from offering advice to clinicians on the investigation, treatment, or counselling of patients. For example, the biochemistry of some rare inherited disorders may be better known to the clinical chemist than to an individual clinician encountering such a case for the first time. In BenGershôm's view ethical considerations should, but rarely do, take precedence over etiquette and should oblige the clinical chemist to participate fully in the clinical decision making process.

With some trepidation the Association of Clinical Biochemists took up the challenge through one of its working parties-the one most concerned with the interaction between clinicians and the laboratory. Instead of attempting to draw up an ethical code of practice on behalf of the association (which everyone realised would be unlikely ever to secure general agreement) the working party drew up a list of questions on ethical matters which were then put to four leaders in clinical chemistry, who were encouraged to answer them purely according to personal conviction. The recorded interviews have now been published, perhaps unfortunately in somewhat ephemeral form (Association of Clinical Biochemists' News Sheet, issues of April to July 1984).

The replies showed a striking degree of concordance. British clinical chemists, whether medically qualified or not, are evidently ready and willing to shoulder the responsibility for facing ethical problems together with the relevant clinician, while unhesitatingly recognising that in case of disagreement the final decision lies with the consultant in charge of the case. All four clinical chemists interviewed regarded coaxing clinicians into using the laboratory more sparingly as an ethical duty. There was less agreement on how strong a stand a chemist should take if he finds himself engaged in a supportive role in clinical research whose ethics seem-usually at some distance and on somewhat flimsy evidence-to be dubious. Attitudes on whether industrial action (especially in support of other groups of staff) is ever justifiable also varied, as in other medical and paramedical professions.

Most of those interviewed were sympathetic to the notion of redeploying to other duties any member of staff who 